\title{
Ex-Vivo Umbilical Cord Blood Expansion
}

National Cancer Institute

\section{Source}

National Cancer Institute. Ex-Vivo Umbilical Cord Blood Expansion. NCI Thesaurus. Code C116665.

The laboratory process of extracting progenitor cells from a given sample of umbilical cord blood and growing them in culture to increase their total quantity prior to transfusion. 